\title{
RECONSTRUCTION OF SOURCE TERMS IN EVOLUTION EQUATIONS BY EXACT CONTROLLABILITY
}

\author{
Masahiro Yamamoto* \\ Department of Mathematical Sciences \\ The University of Tokyo, Japan \\ Komaba, Meguro, Tokyo 153, Japan \\ email: myama@ms.u-tokyo.ac.jp
}

Abstract: For fixed $\rho=\rho(x, t)$, we consider the solution $u(f)$ to

$$
\begin{aligned}
u^{\prime \prime}(x, t)+A u(x, t) & =f(x) \rho(x, t), \quad x \in \Omega, t>0 \\
u(x, 0) & =u^{\prime}(x, 0)=0, \quad x \in \Omega, \\
B_{j} u(x, t) & =0, \quad x \in \partial \Omega, t>0,1 \leq j \leq m,
\end{aligned}
$$

where $u^{\prime}=\frac{\partial u}{\partial t}, u^{\prime \prime}=\frac{\partial^{2} u}{\partial t^{2}}, \Omega \subset R^{r}, r \geq 1$ is a bounded domain with smooth boundary, $A$ is a uniformly symmetric elliptic differential operator of order $2 m$ with $t$-independent smooth coefficients, $B_{j}, 1 \leq j \leq m$, are $t$-independent boundary differential operators such that the system $\left\{A, B_{j}\right\}_{1 \leq j \leq m}$ is wellposed. Let $\left\{C_{j}\right\}_{1 \leq j \leq m}$ be complementary boundary differential operators of $\left\{B_{j}\right\}_{1 \leq j \leq m}$. We consider a multidimensional linear inverse problem : for given $\Gamma \subset \partial \Omega, T>0$ and $n \in\{1, \ldots, m\}$, determine $f(x), x \in \Omega$ from $C_{j} u(f)(x, t)$, $x \in \Gamma, 0<t<T, 1 \leq j \leq n$.

By exact controllability based on the Hilbert Uniqueness Method, we reduce our inverse problem to an equation of the second kind which gives reconstruction of $f$. Moreover under extra regularity assumptions on $\rho$, we can prove that this equation is a Fredholm equation of the second kind. Our methodology is widely applicable to various equations in mathematical physics.

\section{INTRODUCTION}

We consider an initial - boundary value problem :

$$
u^{\prime \prime}(x, t)+A u(x, t)=f(x) \rho(x, t), \quad x \in \Omega, t>0
$$

*This work is partially supported by Sanwa Systems Development Co., Ltd. (Tokyo, Japan). This paper has been written during the author's stay at Weierstraß Institut für Angewandte Analysis und Stochastik in Berlin and he thanks Dr. Johannes Elschner for the invitation.

The original version of this chapter was revised: The copyright line was incorrect. This has been corrected. The Erratum to this chapter is available at DOI: 10.1007/978-0-387-35359-3_40 


$$
\begin{gathered}
u(x, 0)=u^{\prime}(x, 0)=0, \quad x \in \Omega \\
B_{j} u(x, t)=0, \quad x \in \partial \Omega, t>0,1 \leq j \leq m,
\end{gathered}
$$

where $u^{\prime}=\frac{\partial u}{\partial t}, u^{\prime \prime}=\frac{\partial^{2} u}{\partial t^{2}}, \Omega \subset R^{r}, r \geq 1$ is a bounded domain with $C^{2}$ - boundary, $A$ is a uniformly symmetric elliptic differential operator of order $2 m$ with $t$ independent smooth coefficients, $B_{j}, 1 \leq j \leq m$, are boundary differential operators. More precisely, we set $x=\left(x_{1}, \ldots, x_{r}\right) \in R^{r}, \alpha=\left(\alpha_{1}, \ldots, \alpha_{r}\right) \in(N \cup\{0\})^{r}$, $|\alpha|=\alpha_{1}+\ldots+\alpha_{r}, D_{x}^{\alpha}=\left(\frac{\partial}{\partial x_{1}}\right)^{\alpha_{1}} \cdots\left(\frac{\partial}{\partial x_{r}}\right)^{\alpha_{r}}$, and

$$
(A \phi)(x)=\sum_{|\alpha|,|\beta| \leq m}(-1)^{|\alpha|} D_{x}^{\alpha}\left(a_{\alpha \beta}(x) D_{x}^{\beta} \phi\right)(x),
$$

which $a_{\alpha \beta}=a_{\beta \alpha} \in C^{\infty}(\bar{\Omega})$ are real-valued for $|\alpha|,|\beta| \leq m$, and we assume the uniform ellipticity : there exists a constant $M_{0}>0$ independent of $x \in \bar{\Omega}$ and $\xi \in R^{r}$ such that

$$
M_{0}^{-1}|\xi|^{2 m} \leq\left|\sum_{|\alpha|,|\beta|=m} a_{\alpha \beta}(x) \xi^{\alpha+\beta}\right| \leq M_{0}|\xi|^{2 m}, \quad x \in \bar{\Omega}, \xi \in R^{r}
$$

where $\xi=\left(\xi_{1}, \ldots, \xi_{r}\right) \in R^{r}$ and $\xi^{\alpha}=\xi_{1}^{\alpha_{1}} \ldots \xi_{r}^{\alpha_{r}}$ with $\alpha=\left(\alpha_{1}, \ldots, \alpha_{r}\right),|\xi|^{2}=$ $\xi_{1}^{2}+\ldots+\xi_{r}^{2}$. Moreover we put

$$
\left(B_{j} \psi\right)(x)=\sum_{|\alpha| \leq m_{j}} b_{j \alpha}(x) D_{x}^{\alpha} \psi(x)
$$

where $b_{j \alpha} \in C^{\infty}(\partial \Omega), 0 \leq m_{j}<2 m$. Throughout this paper we assume that $\left\{B_{j}\right\}_{1 \leq j \leq m}$ is normal on $\partial \Omega$ (e.g. Lions and Magenes [17] Vol.I) and that the system $\left\{A, B_{j}\right\}_{1 \leq j \leq m}$ is well-posed ([17], Vol.II).

Henceforth let $\left\{\bar{C}_{j}\right\}_{1 \leq j \leq m}$ be complementary boundary differential operators of $\left\{B_{j}\right\}_{1 \leq j \leq m}$, whose coefficients are $t$-independent and smooth in $x \in \partial \Omega($ [17], Vol.I).

In this paper, assuming that $\rho$ is given while $f$ is unknown to be determined from observations on a part of lateral boundary, we denote the weak solution to $(1.1)-(1.3)$ by $u(f)=u(f)(x, t)$. For the weak solution, we can further refer to $[17]$. We discuss

\section{Inverse Source Problem:}

For given $\Gamma \subset \partial \Omega, T>0$ and $n \in\{1, \ldots, m\}$, determine $f(x), x \in \Omega$, from $C_{j} u(f)(x, t), x \in \Gamma, 0<t<T, 1 \leq j \leq n$.

In (1.1), the non-homogeneous term $f(x) \rho(x, t)$ is considered to cause actions such as vibrations, and the inverse source problem is significant in mathematical physics. Moreover when we discuss determination of spatially varying coefficients in $A$, we have to do with this type of inverse problem after subtraction or linearization (e.g. Lavrentiev, Romanov and Shishat-skiı̌[14], Romanov [22]). We notice that we want to determine $f$ with a single boundary measurement. 
In the case where $\rho=\rho(t)$ is independent of $x$, by means of Duhamel's principle (e.g. Rauch [21]), we can reduce the inverse problem to an observability problem, namely, determination of initial data. For the inverse problem in the case of $x$-indepednent $\rho=\rho(t)$, we can refer to Puel and Yamamoto [18], Yamamoto [24], [25], [26]. On the other hand, the inverse problem becomes more difficult for $x$-dependent $\rho$. Fot such a case, the method by Bukhgeim and Klibanov [3] is useful and their method is based on a weighted estimate called a Carleman estimate. For the uniqueness, we can refer to Bukhgeim and Klibanov [3], Iskakov [5], [6], [7], Khaĭdarov [9], Klibanov [10]. Moreover for similar inverse problems for Lamé systems and Maxwell's equations, we refer to Ikehata, Nakamura and Yamamoto [4], and Yamamoto [27], respectively. As for an inverse problem with many observations for a hyperbolic equation given by (1.1), we can refer to Rakesh and Symes [20]. For general references for these kinds of inverse problems, the readers can consult monographs : Isakov [8], Lavrentiev, Romanov and Shishat.skiī[14], Romanov [22].

Most of the papers above-mentioned mainly treat the uniqueness problem. For stability in determining functions in hyperbolic equations from a single boundary measurement, estimation of Hölder type has been proved (Khaĭdarov [9]. also see a remark (p.577) in [10]). Recently the author has established the best possible Lipschitz stability by combination of the Carleman estimate and the exact observability (Yamamoto [28]).

Reconstruction of $f$ is practically important, but such discussions are very few (Bukhgeim [2]). The purpose of this paper is to reduce our inverse problem to an equation of the second kind by the exact controllability, which is a Fredholm equation of the second kind under a natural setting. Then our inverse problem is to solve the equation of the second kind. Further study for the equation will be made in a forthcoming paper.

This paper is composed of four sections. Section 2 is devoted to a brief explanation of the Hilbert Uniqueness Method. In Section 3, we state our main result. In Section 4, we prove the main result.

\section{BRIEF EXPLANATION OF THE HILBERT UNIQUENESS METHOD}

We give a brief explanation of the Hilbert Uniqueness Method, according to Lions [16]. We refer also to Komornik [11], Lasiecka and Triggiani [13], Lions [15]. We set

$$
\begin{aligned}
\widetilde{F} & =\widetilde{F_{1}} \times \widetilde{F_{2}} \\
& =\left\{\left(\phi_{1}, \phi_{2}\right) \in C^{\infty}(\bar{\Omega})^{2} ; B_{j} \phi_{1}=0 \text { if the order of } B_{j} \text { is less than } m\right\},
\end{aligned}
$$

and for $\left(\phi_{1}, \phi_{2}\right) \in \widetilde{F}$, we denote the solution to

$$
\begin{aligned}
& w^{\prime \prime}(x, t)+A w(x, t)=0, \quad x \in \Omega, 0<t<T, \\
& w(x, 0)=\phi_{1}(x), \quad w^{\prime}(x, 0)=\phi_{2}(x), \quad x \in \Omega
\end{aligned}
$$




$$
B_{j} w(x, t)=0, \quad x \in \partial \Omega, 0<t<T, 1 \leq j \leq m
$$

by $w\left(\phi_{1}, \phi_{2}\right)=w\left(\phi_{1}, \phi_{2}\right)(x, t)$. We pose

Assumption A (Unicity)

For a given measurable $\Gamma \subset \partial \Omega$, a finite $T>0$ and $n \in\{1, \ldots, m\}$, if the solution $w\left(\phi_{1}, \phi_{2}\right)$ satisfies

$$
C_{j} w(x, t)=0, \quad x \in \Gamma, 0<t<T, 1 \leq j \leq n
$$

for $\left(\phi_{1}, \phi_{2}\right) \in \widetilde{F}$, then $w\left(\phi_{1}, \phi_{2}\right)(x, t)=0, x \in \Omega, 0<t<T$ follows.

This is unicity in a Cauchy problem for $w^{\prime \prime}+A w=0$, for which we refer to Bardos, Lebeau and Rauch [1] and Tataru [23] for example. On Assumption A, we can define a norm $\left\|\left(\phi_{1}, \phi_{2}\right)\right\|_{F}$ by

$$
\left\|\left(\phi_{1}, \phi_{2}\right)\right\|_{F} \equiv\left(\left\|\phi_{1}\right\|_{F_{1}}^{2}+\left\|\phi_{2}\right\|_{F_{2}}^{2}\right)^{\frac{1}{2}}=\left(\sum_{j=1}^{n}\left\|C_{j} w\left(\phi_{1}, \phi_{2}\right)\right\|_{L^{2}(\Gamma \times(0, T))}^{2}\right)^{\frac{1}{2}}
$$

for any $\left(\phi_{1}, \phi_{2}\right) \in \tilde{F}$, where $\|\eta\|_{L^{2}(\Gamma \times(0, T))}=\left(\int_{\Gamma} \int_{0}^{T}|\eta(x, t)|^{2} d t d S_{x}\right)^{\frac{1}{2}}$. Let a Hilbert space $F \equiv F_{1} \times F_{2}$ be the completion of $\tilde{F}$ by the norm $\|\cdot\|_{F}$. Let $F^{\prime}=F_{1}^{\prime} \times F_{2}^{\prime}$ be its dual. Throughout this paper,.$^{\prime}$ denotes the dual space and we identify the dual spaces $L^{2}(\Gamma \times(0, T))^{\prime}$ of $L^{2}(\Gamma \times(0, T))$ and $L^{2}(\Omega)^{\prime}$ of $L^{2}(\Omega)$ respectively with itself. The space $F^{\prime}$ is related to the exactly controllable set and the essence of the Hilbert Uniqueness Method is construction of the Hilbert space $F^{\prime}$.

Next let us consider

$$
\begin{gathered}
\psi^{\prime \prime}(x, t)+A \psi(x, t)=0, \quad x \in \Omega, 0<t<T \\
\psi(x, T)=\psi^{\prime}(x, T)=0, \quad x \in \Omega \\
B_{j} \psi(x, t)= \begin{cases}v_{j}(x, t), & x \in \Gamma, 0<t<T: 1 \leq j \leq n \\
0, & x \in \partial \Omega \backslash \Gamma, 0<t<T: 1 \leq j \leq n \\
0, & x \in \partial \Omega, 0<t<T: n+1 \leq j \leq m .\end{cases}
\end{gathered}
$$

For the system (2.4) - (2.6) with a uniformly symmetric elliptic operator $A$ of order $2 m$, a general treatment (Theorem 4.1 (p.107: Vol.II) in [17]) tells that for any $v=\left(v_{1}, \ldots, v_{n}\right) \in L^{2}(\Gamma \times(0, T))^{n}$, there exists a unique weak solution $\psi(v) \in H^{0,-1}(\Omega \times(0, T)) \equiv\left(H_{0}^{1}\left(0, T ; L^{2}(\Omega)\right) \cap L^{2}\left(0, T ; L^{2}(\Omega)\right)\right)^{\prime}$, where $H_{0}^{1}\left(0, T ; L^{2}(\Omega)\right)=\left\{u \in H^{1}\left(0, T ; L^{2}(\Omega)\right) ; u(\cdot, 0)=u(\cdot, T)=0\right\}$. Furthermore we refer to Theorems 6.1 and 6.2 (pp.118-119: Vol.II) in [17], and especially 
for a wave equation, we also quote Lasiecka, Lions and Triggiani [12], Lions [16].

In applying a result (Theorem 0 below) on exact controllability, we however pose a stronger assumption for the regularity of $\psi(v)$.

Assumption B (Regularity in the control system)

For $v \in L^{2}(\Gamma \times(0, T))^{n}$, the weak solution $\psi(v)$ satisfies

$$
\begin{gathered}
\psi(v) \in C^{0}\left([0, T] ; F_{2}^{\prime}\right), \quad \psi(v)^{\prime} \in C^{0}\left([0, T] ; F_{1}^{\prime}\right) \\
\|\psi(v)\|_{C^{0}\left([0, T] ; F_{2}^{\prime}\right)} \leq M_{1}\|v\|_{L^{2}(\Gamma \times(0, T))^{n}}
\end{gathered}
$$

where $M_{1}=M_{1}(\Omega, \Gamma, T)>0$ is independent of $v$.

Example 1 : wave equation ([11], [13], [16])

For an arbitrarily given $x_{0} \in R^{r}$, we set

$$
\begin{aligned}
\Gamma_{+}\left(x_{0}\right) & =\left\{x \in \partial \Omega ;\left(x-x_{0}, \nu(x)\right)>0\right\} \\
R_{0} & =R_{0}\left(x_{0}\right)=\sup _{x \in \partial \Omega}\left|x-x_{0}\right|,
\end{aligned}
$$

where $\nu(x)$ is the outward unit normal to $\partial \Omega$ and $(\cdot, \cdot)$ is the inner product in $R^{r}$. We consider: $A=-\Delta$ (the Laplacian), $m=1$,

$$
B_{1} u=\left.u\right|_{\partial \Omega}, \quad C_{1} u=\frac{\partial u}{\partial n \mid \Gamma} .
$$

If

$$
T>2 R_{0}
$$

and a measurable set $\Gamma \subset \partial \Omega$ satisfies

$$
\Gamma \supset \Gamma_{+}\left(x_{0}\right)
$$

then

$$
F_{1}=H_{0}^{1}(\Omega), \quad F_{2}=L^{2}(\Omega),
$$

and Assumptions A and B hold true.

Example II: plate equation (e.g. [11], [16]).

Let $A=\Delta^{2}, m=2$ and

$$
B_{1} u=\left.u\right|_{\partial \Omega}, \quad B_{2} u=\frac{\partial u}{\partial n}, \quad C_{1} u=\Delta u_{\mid \Gamma}, \quad C_{2} u=\frac{\partial \Delta u}{\partial n} \mid \Gamma .
$$

We set $n=1$. If we choose $\Gamma$ satisfying (2.8), then for any $T>0, F_{2}=L^{2}(\Omega)$ holds, and Assumptions A and B hold true.

By the Hilbert Uniqueness Method, we show boundary exact controllability: Theorem 0 (Théorème 3.2 (p.119) in [16]) On Assumptions A and B, for any $\left(\phi_{1}, \phi_{2}\right) \in F_{2}^{\prime} \times F_{1}^{\prime}$, there exists $v=\left(v_{1}, \ldots, v_{n}\right) \in L^{2}(\Gamma \times(0, T))^{n}$ such that the weak solution $\psi=\psi(v)$ to (2.4) - (2.6) satisfies

$$
\psi(v)(\cdot, 0)=\phi_{1}, \quad \psi(v)^{\prime}(\cdot, 0)=\phi_{2} .
$$


Moreover we can construct a map from $\left(\phi_{1}, \phi_{2}\right)$ to $v$ such that

$$
\|v\|_{L^{2}(\Gamma \times(0, T))^{n}} \leq M_{1}\left(\left\|\phi_{1}\right\|_{F_{2}^{\prime}}+\left\|\phi_{2}\right\|_{F_{1}^{\prime}}\right), \quad\left(\phi_{1}, \phi_{2}\right) \in F_{2}^{\prime} \times F_{1}^{\prime},
$$

where $M_{1}=M_{1}(\Omega, \Gamma, T)>0$ is independent of $\left(\phi_{1}, \phi_{2}\right)$.

This theorem defines a bounded linear operator $g: F_{2}^{\prime} \longrightarrow L^{2}(\Gamma \times(0, T))^{n}$ which maps $\phi_{1} \in F_{2}^{\prime}$ to $v \in L^{2}(\Gamma \times(0, T))^{n}$ realizing $\psi(v)(\cdot, 0)=\phi_{1}$ and $\psi(v)^{\prime}(\cdot, 0)=0$, and

$$
\left\|g\left(\phi_{1}\right)\right\|_{L^{2}(\Gamma \times(0, T))^{n}} \leq M_{1}\left\|\phi_{1}\right\|_{F_{2}^{\prime}} .
$$

In (2.6), $v_{j}, 1 \leq j \leq n$, are regarded as boundary controls which steer the system described by (2.4) - (2.5) to the equilibrium at time $\mathrm{T}$ starting from the initial state given by $\left(\phi_{1}, \phi_{2}\right)$.

\section{MAIN RESULT: REDUCTION OF THE GENERAL INVERSE SOURCE PROBLEM TO AN EQUATION OF THE SECOND KIND}

We discuss the initial - boundary value problem $(1.1)-(1.3)$ with $\rho=\rho(x, t)$ satisfying

$$
\begin{gathered}
\left\|\int_{0}^{T} \rho^{\prime}(\cdot, t) \psi(\cdot, t) d t\right\|_{F_{2}^{\prime}} \leq M_{2}\|\psi\|_{C^{0}\left([0, T] ; F_{2}^{\prime}\right)}, \quad \psi \in C^{0}\left([0, T] ; F_{2}^{\prime}\right) \\
\|f \rho(\cdot, 0)\|_{F_{2}} \leq M_{2}\|f\|_{F_{2}}, \quad f \in F_{2} \\
\rho \in H^{1}\left(0, T ; L^{\infty}(\Omega)\right) \\
\left\|f \rho^{\prime}\right\|_{L^{2}\left(0, T ; F_{2}\right)} \leq M_{2}\|f\|_{F_{2}}, \quad f \in F_{2}
\end{gathered}
$$

Here $M_{2}>0$ is independent of $\psi$ and $f$. We always pose Assumptions $\mathrm{A}$ and B.

Remark If we can characterize $F_{2}$, for example, as $F_{2}=L^{2}(\Omega)$ (cf. Examples in Section 2), then the conditions (3.1) - (3.4) are equivalent to

$$
\rho \in H^{1}\left(0, T ; L^{\infty}(\Omega)\right), \quad \rho(\cdot, 0) \in L^{\infty}(\Omega) .
$$

We recall that a linear operator $g: F_{2}^{\prime} \longrightarrow L^{2}(\Gamma \times(0, T))^{n}$ is defined in Theorem 0 in Section 2 and satisfies (2.11). We define a linear operator $S$ in $F_{2}^{\prime}$ by

$$
\left(S \phi_{1}\right)(x)=\int_{0}^{T} \rho^{\prime}(x, t) \psi\left(g\left(\phi_{1}\right)\right)(x, t) d t, \quad \phi_{1} \in F_{2}^{\prime} .
$$

Then we are ready to state the main result: 
Theorem Under Assumptions A and B, (3.1) - (3.4);

(1) $S: F_{2}^{\prime} \longrightarrow F_{2}^{\prime}$ is a bounded linear operator.

(2) Let $v \in H^{1}\left(0, T ; L^{2}(\Gamma)\right)^{n}$. Then $f \in F_{2}$ satisfies

$$
g^{*}\left(v^{\prime}-\left(C_{1} u(f)^{\prime}, \ldots, C_{n} u(f)^{\prime}\right)\right)=0
$$

if and only if $f \in F_{2}$ satisfies

$$
\rho(\cdot, 0) f+S^{*} f=g^{*} v^{\prime} .
$$

Here $S^{*}: F_{2} \longrightarrow F_{2}$ is the adjoint of $S: F_{2}^{\prime} \longrightarrow F_{2}^{\prime}$, and $g^{*}$ is the one of a bounded linear operator $g: F_{2}^{\prime} \longrightarrow L^{2}(\Gamma \times(0, T))^{n}$. The operator equation (3.7) is our desired one of the second kind.

Corollary 3.1 If $f$ is a solution of our inverse problem, that is, $f \in F_{2}$ satisfies

$$
\left(C_{1} u(f), \ldots, C_{n} u(f)\right)=v
$$

for $v \in H^{1}\left(0, T ; L^{2}(\Gamma)\right)^{n}$, then $f$ solves (3.7).

Remark In general, $\mathcal{R}(g)$ is not dense in $L^{2}(\Gamma \times(0, T))^{n}$, so that $g^{*}$ is not injective. Thus in Theorem, we can not replace (3.6) by $\left(3.6^{\prime}\right)$.

Henceforth we assume

$$
\rho(x, 0) \neq 0, \quad x \in \bar{\Omega} .
$$

Then (3.7) is an equation of the second kind:

$$
f+\frac{1}{\rho(\cdot, 0)} S^{*} f=\frac{1}{\rho(\cdot, 0)} g^{*} v^{\prime}
$$

Moreover Corollary 1 asserts that it is sufficient to consider (3.9) for reconstructing $f$. For similar linear inverse problems with singular data such as Dirac delta functions in multidimensional cases and similar ones with smooth data in one-dimensional cases, we can reduce the problems to a Volterra equation of the second kind (e.g. Chapter 2 and Section 3 of Chapter 4 in [22]). However in multidimensional cases with not necessarily singular data, a general way for such reduction has not been published (cf. Bukhgeim [2]).

Here we do not give direct expression of $S^{*}$. In special cases, direct expression of $S^{*}$ is not difficult. For example, in Example 1 in Section 2, let $r=1$ (i.e., the spatial dimension is 1 ), $\Omega=(0,1), \Gamma=\{0\}$ (one end point) and $T=2$. Then we can construct the control operator $g: L^{2}(0,1) \longrightarrow L^{2}(0,2)$ by consideration of the dependency domain of the one-dimensional wave equation and D'Alembert's formula.

Next we have to study the unique solvability of the equation (3.9). First by the contraction mapping principle, we can readily see 


\section{Corollary 3.2 Let}

$$
\left\|\frac{\rho^{\prime}(\cdot, \cdot)}{\rho(\cdot, 0)}\right\|_{L^{1}\left(0, T ; L^{\infty}(\Omega)\right)}
$$

be sufficiently small and let $v=\left(C_{1} u(f), \ldots, C_{n} u(f)\right)$. Then $f$ is given as a unique solution of (3.9) by iteration.

We consider a hyperbolic equation of the second order and we take $C_{1} u=$ $\left.\frac{\partial u}{\partial n}\right|_{\Gamma}$ as the boundary observation where the subboundary $\Gamma$ satisfies (2.8):

$$
\begin{gathered}
u^{\prime \prime}(x, t)=\Delta u(x, t)-p(x) u(x, t)+f(x) \rho(x, t), \quad x \in \Omega, t>0 \\
u(x, 0)=u^{\prime}(x, 0)=0, \quad x \in \Omega \\
u(x, t)=0, \quad x \in \partial \Omega, t>0 .
\end{gathered}
$$

Moreover in addition to (3.1') we assume

$$
\begin{gathered}
p \in L^{\infty}(\Omega) \\
\rho, \frac{\rho}{\rho(\cdot, 0)} \in H^{2}\left(0, T ; L^{\infty}(\Omega)\right)
\end{gathered}
$$

$$
T>2 R_{0}
$$

where $R_{0}$ is given by (2.7). Then by the argument in the proof of Lemma 5.5 in Puel and Yamamoto [19], we can prove

Corollary 3.3 Under the assumptions (3.14) - (3.16), the operator $S^{*}: L^{2}(\Omega) \longrightarrow$ $L^{2}(\Omega)$ is compact. Therefore the equation (3.9) is a Fredholm equation of the second kind in $L^{2}(\Omega)$.

In Corollary 3 , for the unique solvability, it suffices to verify that $f+\frac{1}{\rho(\cdot, 0)} S^{*} f=$ 0 implies $f=0$. This is equivalent to the uniqueness in some inverse problem and the method in Bukhgeim and Klibanov [3] may be helpful. In a forthcoming paper, we will treat details of the unique solvability.

\section{References}

[1] Bardos, C., Lebeau, G. and Rauch, J., Sharp sufficient conditions for the observation, control, and stabilization of waves from the boundary. SIAM J. Control and Optimization V.30 (1992), 1024 - 1065.

[2] Bukhgeim, A.L., private communication. 
[3] Bukhgeim, A.L. and Klibanov, M.V., Global uniqueness of a class of multidimensional inverse problems. (English translation) Soviet Math. Dokl. V.24 (1981), 244-247.

[4] Ikehata, M., Nakamura, G. and Yamamoto, M., Uniqueness in inverse problems for the isotropic Lamé system. to appear in Journal of Mathematical Sciences of The University of Tokyo (1998).

[5] Isakov, V., A nonhyperbolic Cauchy problem for $\square_{b} \square_{c}$ and its applications to elasticity theory. Comm. Pure and Applied Math. V.34 (1986), 747-767.

[6] Isakov, V., Inverse Source Problems. American Mathematical Society, Providence, Rhode Island, 1990

[7] Isakov, V., Uniqueness and stability in multi-dimensional inverse problems. Inverse Problems V.9 (1993), 579-621.

[8] Isakov, V., Inverse Problems for Partial Differential Equations. SpringerVerlag, Berlin 1998

[9] Khắdarov, A., On stability estimates in multidimensional inverse problems for differential equations. aperinfo (English translation) Soviet Math. Dokl. V.38 (1989), 614-617.

[10] Klibanov, M.V., Inverse problems and Carleman estimates. Inverse Problems V.8 (1992), 575-596.

[11] Komornik, V., Exact Controllability and Stabilization : the Multiplier Method. John Wiley \& Sons, Chichester 1994.

[12] Lasiecka, I., Lions, J.-L. and Triggiani, R., Non homogeneous boundary value problems for second order hyperbolic operators. J. Math. Pures Appl. V.65 (1986), 149-192.

[13] Lasiecka, I. and Triggiani, R., Exact controllability of the wave equation with Neumann boundary control. Appl. Math. Optim. V.19 (1989), 243290.

[14] Lavrent'ev, M.M., Romanov, V.G. and Shishat·skiŭ, S.P., Ill-posed Problems of Mathematical Physics and Analysis. American Mathematical Society, Providence, Rhode Island (English translation) 1986.

[15] Lions, J.-L., Exact controllability, stabilization and perturbations for distributed systems. SIAM Review V.30 (1988), 1-68.

[16] Lions, J.-L., Contrôlabilité Exacte Perturbations et Stabilisation de Systèmes Distribués. Vol.1. Masson, Paris 1988.

[17] Lions, J.-L. and Magenes, E., Non-homogeneous Boundary Value Problems and Applications. Volumes I and II. (English translation) Springer-Verlag, Berlin 1972.

[18] Puel, J.-P. and Yamamoto, M., Applications de la contrôlabilité exacte à quelques problèmes inverses hyperboliques. C. R. Acad. Sci. Paris Sér.I Math. V.320 (1995), 1171-1176. 
[19] Puel, J.-P. and Yamamoto, M., Generic well-posedness in a multidimensional hyperbolic inverse problem. Journal of Inverse and Ill-posed Problems V.5 (1997), 55-83.

[20] Rakesh and Symes, W.W., Uniqueness for an inverse problem for the wave equation. Commun. in Partial Differential Equations V.13 (1988), 87-96.

[21] Rauch, J., Partial Differential Equations. Springer-Verlag, Berlin 1991.

[22] Romanov, V.G., Inverse Problems of Mathematical Physics. (English translation) VNU Science Press, Utrecht 1987

[23] Tataru, D., Unique continuation for solutions to PDE's; between Hörmander and Holmgren's theorems. Commun. in Partial Differential Equations V. 20 (1995), 855-884.

[24] Yamamoto, M., Stability, reconstruction formula and regularization for an inverse source hyperbolic problem by a control method. Inverse Problems V.11 (1995), 481-496.

[25] Yamamoto. M., Application of the Hilbert uniqueness method to inverse source problems: stability and regularization. Z. Angew. Math. Mech. V.75, Suppl. 2 (1995), 515-516.

[26] Yamamoto, M., On ill-posedness and a Tikhonov regularization for a multidimensional inverse hyperbolic problem. J. Math. Kyoto University V.36 (1996), 825-856.

[27] Yamamoto, M., On an inverse problem of determining source terms in Maxwell's equations with a single measurement. in "Inverse Problems, Tomography and Image Processing", edited by A.G. Ramm, Plenum Press, New York (1998), 241-256

[28] Yamamoto, M., Uniqueness and stability in multidimensional hyperbolic inverse problems. to appear in J. Math. Pures Appl. (1998). 